\title{
End-to-End Throughput Analysis of Multi-Hop Wireless Networks Using Stochastic Geometry
}

\author{
Yuan Liang \\ liangy11@egr.msu.edu \\ Michigan State University \\ Department of Electrical \& Computer \\ Engineering \\ East Lansing, MI 48824, USA
}

\author{
Jian Ren \\ renjian@egr.msu.edu \\ Michigan State University \\ Department of Electrical \& Computer \\ Engineering \\ East Lansing, MI 48824, USA
}

\author{
Tongtong Li \\ tongli@egr.msu.edu \\ Michigan State University \\ Department of Electrical \& Computer \\ Engineering \\ East Lansing, MI 48824, USA
}

\begin{abstract}
This paper investigates the effect of relay randomness on the endto-end throughput in multi-hop wireless networks using stochastic geometry. We model the nodes as Poisson Point Processes and calculate the spatial average of the throughput over all potential geometrical patterns of the nodes, with no constraints on the relay density or routing distance. More specifically, first, assuming nearest neighbor routing protocol, we derive the distribution of the longest hop distance in a multi-hop route for any given routing distance. Second, we analyze the average end-to-end throughput under the TDMA protocol. Our analysis indicates that compared with the relay-free case, even randomly distributed relays can significantly extend the communication distance. It is also observed that systems with equidistant relays generally achieve much higher throughput than those with random relays. Moreover, the optimal relay intensity varies with the routing distance, node density and interference levels. Our results are demonstrated through numerical examples.
\end{abstract}

\section{CCS CONCEPTS}

- Networks $\rightarrow$ Network performance modeling; Network performance analysis; • General and reference $\rightarrow$ Performance; - Computer systems organization $\rightarrow$ Fault-tolerant network topologies;

\section{KEYWORDS}

end-to-end throughput, stochastic geometry, Poisson point process

\section{INTRODUCTION}

Multi-hop communication with relay assistance has become a prominent scheme in today's hybrid network design. The main reason is that it can extend the communication distance in wireless networks without the deployment of wired backhaul facilities. In wireless networks, the geometric locations of the nodes play a key role in determining the signal to interference and noise ratio (SINR), and hence the probability of successful transmission, at each receiver.

Permission to make digital or hard copies of all or part of this work for are not made or distributed for profit or commercial advantage and that copies bear this notice and the full citation on the first page. To copy otherwise, to republish, to post on servers or to redistribute to lists, requires prior specific permission and/or a fee.

MOBIMEDIA 2017, July 13-14, Chongqing, People's Republic of China Copyright @ 2017 EAI 978-1-63190-156-0
In large scale multi-hop wireless networks, the node locations, including the relay locations, are generally random. The spatial randomness in node locations raises significant challenges in network performance analysis.

An effective tool in dealing with spatial randomness in wireless networks is stochastic geometry, for which the basic idea is to model the nodes as Poisson Point Processes (PPPs) and calculate the spatial averages of network performance characteristics by averaging over all potential geometrical patterns of the nodes [1, 7, 9-11].

In literature, stochastic geometry modeling has been utilized to study multi-hop wireless network. In [2][16], the random access transport capacity and the end-to-end delay were evaluated respectively. In [4][14][17], the single-hop performance metrics in multi-hop wireless networks were studied. In [8], the authors introduced limited random deviations of relays from their ideal locations in the equidistant deployment. In most of the existing works on the end-to-end performance analysis, the locations of relays are assumed to be deterministic and known, and are often approximated as equidistant. Even though the results obtained provide good approximation of random relays if the relay intensity is dense enough and certain routing protocols are employed, the randomness of relays is not fully characterized and taken into consideration.

In this paper, we analyze the end-to end throughput of a general multi-hop route in a wireless network with randomly located relays. In our analysis, we model the relays as a linear PPP between the source and destination. Under the nearest neighbor $(\mathrm{NN})$ routing protocol, we evaluate the end-to-end throughput under a TDMA protocol. The external interferers are modeled as an independent PPP over the whole plane, following the ALOHA MAC protocol. Our model is similar to that of [5], however, our work studies the end-to-end throughput that was not formally addressed before. We derive the distribution of the longest hop distance in a multi-hop route and calculate the average end-to-end throughput. Our numerical results, together with the theoretical analysis, indicates that compared with the relay-free case, the communication distance between source and destination can generally be extended using random relays, even without a fine deployment. It is also observed that compared with the equidistant relays, the end-to-end throughput under random relays suffers a significant performance loss, which is not negligible in network performance evaluation. Moreover, optimal relay intensities can be calculated for different route distances. If the intensity of the available relays is greater than the optimal value, the actual relays can be selected from a thinning of the original relay point process to get a better performance. 


\section{SYSTEM DESCRIPTION}

\subsection{Network Model}

We consider a source node $S$, and a destination node $D$ located at a distance of $R$. A one-dimensional linear relay pattern is studied, where relay nodes are distributed randomly along the line segment between $S$ and $D$. Without loss of generality, we assume $S$ is at the origin and $D=(R, 0)$. Thus the relays formulate a $1 \mathrm{D}$ point process $\Phi=\left\{X_{i}, i=1,2, \ldots, N\right\}$, where $N$ is the random variable (RV) denoting the number of relays, and $X_{i}$ the location of the $i$-th relay along the line segment between $(0,0)$ and $(R, 0)$. In the remaining part of this paper, we model $\Phi$ as a $1 \mathrm{D}$ homogeneous PPP (HPPP) of intensity $\lambda$, i.e., for $i=0,1, \ldots, N-1$ and let $X_{0}=S$. The hop distances $L_{i}=\left|X_{i+1}-X_{i}\right|$ are exponentially distributed independent RVs of mean $1 / \lambda$ [5]. The locations of the relays would remain static, in contrast to the high mobility model. Considering a backlogged source $S$ which has infinite packets to transmit, we define the end-to-end throughput from $S$ to $D$ as the number of packets initiated from source $S$ that are successfully received at destination $D$ per time slot.

The nearest neighbor routing protocol is employed, where relay node $X_{i}$ would transmit the packets originated from the source $S$ to its nearest neighbor $X_{i+1}$ in the direction to $D$ in a decode-andforward manner. For tractable analysis, we assume that each relay node has an infinite transmission buffer, and each packet relayed is served in a first-in first-out fashion. The packet that fails in one transmission would go back to the head of the transmission queue, waiting for the opportunity of next transmission. We consider a TDMA MAC protocol for the relay nodes. Given there are $N$ relay nodes, a TDMA cycle would consist of $N+1$ time slots, each of which would be assigned to one relay node or the source node. Since only one node on the route would be allowed to transmit signals at each time slot, no intra-route inference is introduced.

We apply the decoupling technique in [5] to our network model, where all the other nodes that are not along the $S$ - $D$ path are modeled as an independent 2 D point process $\Psi$ over $\mathbb{R}^{2}$ from $\Phi$. Potentially, these nodes can be the external interferers to the relays we study when they transmit over the same spectrum and time slot. For the remaining part of this paper, we model $\Psi$ as a 2D HPPP of intensity $\mu$. We assume that the transmissions of the nodes in $\Psi$ follow the ALOHA protocol, where each node would transmit at each time slot independently with a probability of $p_{a}$.

As can be seen, the network model adopted here is actually a combination of the models in [15] and [5]. More specifically, we combine the random relay model in [5] with the TDMA/ALOHA multi-hop network model in [15].

\subsection{Channel Model}

Both large-scale path-loss and small-scale fading are considered. The received power of a signal transmitted at a distance of $x$ meters with transmitting power $P_{T}$ is

$$
P_{R}(x)=\frac{P_{T} \cdot H}{c \cdot x^{\beta}}
$$

where $H$ is a random channel gain, $\beta$ is the path-loss exponent, and $c$ is a constant determined by the antenna gains and signal wavelength. $H$ is an exponentially distributed random variable with mean 1, i.e., Rayleigh fading is considered. Independent small scale fading is assumed for different transmitter-receiver pairs in different time slots. The small scale fading between two locations $\boldsymbol{x}_{1}$ and $\boldsymbol{x}_{2}$ is represented by $H_{\boldsymbol{x}_{1}, \boldsymbol{x}_{2}}$.

\subsection{SINR Characterization}

Assume that the physical layer uses a fixed rate coding scheme, where a packet can be successfully received iff the received signal to interference and noise ratio (SINR) is above a given threshold $\theta$. We consider an interference-limiting scenario, where the noise power is negligible compared with the interference power, so we use signal to interference ratio (SIR) and SINR interchangeably. Without loss of generality, we assume that each node in the network transmits with unit power. For $i=1,2, \ldots, N+1$ and let $X_{N+1}=D$, the received SIR at relay $X_{i}$ can be expressed by

$$
\operatorname{SIR}\left(X_{i}\right)=\frac{H_{X_{i-1}, X_{i}}\left|X_{i}-X_{i-1}\right|^{-\beta}}{\sum_{Y_{j} \in \Psi} B\left(Y_{j}\right) H_{Y_{j}, X_{i}}\left|Y_{j}-X_{i}\right|^{-\beta}},
$$

where for any $Y_{j} \in \Psi$, the binary $\mathrm{RV} B\left(Y_{j}\right)$ indicates whether the "external" node $Y_{j}$ would transmit under ALOHA protocol at the time slot of interest. The coverage probability at $X_{i}$ is $\operatorname{Pr}\left\{\operatorname{SIR}\left(X_{i}\right)>\right.$ $\theta\}$.

The distribution of external interferers in a given time slot can be viewed as an independent thinning of $\Psi$ with a retention probability of $p_{a}$, i.e., an HPPP with intensity $\mu^{\prime}=p_{a} \mu$. Following the same assumption made in [15], we make the approximation that packet successes are independent across different hops and time slots, i.e., the distribution of external interferers are independent across different time slots. Under such approximation, the coverage probability for an individual hop of distance $l$ can be calculated as follows.

Lemma 2.1. Given a hop distance l, the coverage probability for the hop is

$$
\begin{aligned}
& \qquad P_{S}(l)=\exp \left(-\kappa l^{2}\right), \\
& \text { where } \kappa=2 \pi \mu^{\prime} \frac{\pi}{\beta \sin (2 \pi / \beta)} \theta^{2 / \beta} .
\end{aligned}
$$

Proof. See [4].

Remark 1. For the tractability of the problem, in this paper, we only consider the case where relays are deployed along the line segment between $S$ and $D$. However, the results obtained also apply to the more practical scenario where relays are modeled as a $2 \mathrm{D}$ HPPP over an area. For example, in Fig. 1, the relays are deployed randomly in a $R \times W$ rectangle $\mathcal{R}$ whose widths intersect $S$ and $D$. A simple NN routing protocol is that each relay would transmit to its nearest neighbor along the direction to $D$ (the $x$-coordinate). By projecting the relays to the $x$-coordinate, we can find that the hop distances in the 2D case are lower bounded by the hop distances in the $1 \mathrm{D}$ case. Thus, the throughput in $1 \mathrm{D}$ case is an upper bound of the throughput in $2 \mathrm{D}$ case.

\subsection{Problem Formulation}

The local throughput at node $X_{i}$ on the route can be calculated by

$$
T_{\text {local }}\left(X_{i}\right)=\frac{1}{N+1} P_{S}\left(\left|X_{i}-X_{i-1}\right|\right)
$$




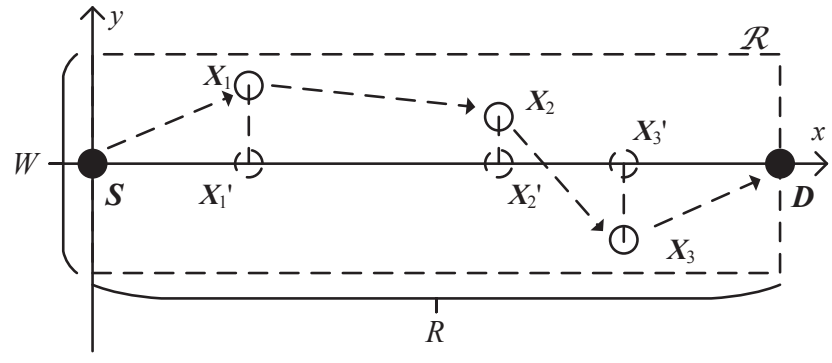

Figure 1: An illustration of relays randomly deployed over a $2 \mathrm{D}$ area

where $P_{S}\left(\left|X_{i}-X_{i-1}\right|\right)$ is the coverage probability or the probability of successful transmission from $X_{i-1}$ to $X_{i}$. According to the stability analysis in queuing theory, the end-to-end throughput is bounded by the hop of the lowest throughput [13]. Since $P_{S}(\cdot)$ is a non-increasing function, the end-to-end throughput can be expressed as

$$
T_{\text {end }}=\min _{\boldsymbol{X}_{i} \in \Phi \cup\{\boldsymbol{D}\}} T_{\text {local }}\left(\boldsymbol{X}_{i}\right)=\frac{1}{N+1} P_{s}\left(\max _{\boldsymbol{X}_{i} \in \Phi \cup\{\boldsymbol{D}\}}\left|\boldsymbol{X}_{i}-\boldsymbol{X}_{i-1}\right|\right) .
$$

Denote the longest hop distance on the route by $L_{m}$, then we have

$$
T_{\text {end }}=\frac{1}{N+1} P_{S}\left(L_{m}\right) .
$$

Note that $N$ and $L_{m}$ are not independent, the distribution of $N$ and $L_{m}$ are determined by the source-destination distance $R$ and the relay distribution. In the following, we will show how to calculate the average end-to-end throughput $\mathbb{E}\left\{T_{\text {end }}\right\}$. We first start with the distribution of $L_{m}$.

\section{THE DISTRIBUTION OF THE LONGEST HOP DISTANCE $L_{M}$}

In this section, we derive the distribution of the longest hop distance $L_{m}$, as well as its mean and variance.

THEOREM 3.1. Given the routing distance between source and destination $R=r$, we have:

(1) The conditional CDF of $L_{m}, \operatorname{Pr}\left\{L_{m} \leq l \mid R=r\right\}=1$ for $l \geq r$. Moreover $\operatorname{Pr}\left\{L_{m}=r \mid R=r\right\}=e^{-\lambda r}$ and $\operatorname{Pr}\left\{L_{m}<r \mid R=\right.$ $r\}=1-e^{-\lambda r}$.

(2) Define $g(l, r) \triangleq \operatorname{Pr}\left\{L_{m} \leq l \mid R=r\right\}$ and denote the Laplace transform $(L T)$ of $g(l, r)$ with respect to $r$ by $G(l, s)$, then

$$
G(l, s)=\frac{1-e^{-(\lambda+s) l}}{s+\lambda e^{-(\lambda+s) l}} .
$$

Proof. 1) This part follows directly from the properties of PPP.

2) For $0<l<r$, consider the conditional probability of $L_{m}$ given that the first relay $X_{1}$ is located at $(x, 0), \operatorname{Pr}\left\{L_{m} \leq l|R=r,| X_{1} \mid=x\right\}$. Since the distribution of the points of $\Phi$ in disjoint intervals are independent, and based on the Palm theory of $\mathrm{PPP}^{1}$, given $\left|X_{1}\right|=x$,

\footnotetext{
${ }^{1}$ For a PPP, given that one node is located at a particular point, the conditional distribution of all other nodes is still a PPP, which is known as Slivnyak-Mecke Theorem [3, Theorem 1.4.5].
}

the remaining relay nodes within the interval $(x, r)$ is still a $1 \mathrm{D}$ PPP of the same intensity with $\Phi$. And the distribution of the longest hop distance for the relays within $(x, r)$ should be the same as that for $R=r-x$. Since $\left|X_{1}\right|$ is exponentially distributed for $x<r$, we have

$$
\begin{aligned}
\operatorname{Pr}\left\{L_{m} \leq l \mid R=r\right\} & =\int_{0}^{l} f_{\left|X_{1}\right|}(x) \operatorname{Pr}\left\{L_{m} \leq l|R=r,| X_{1} \mid=x\right\} \mathrm{d} x \\
& =\int_{0}^{l} \lambda e^{-\lambda x} \operatorname{Pr}\left\{L_{m} \leq l \mid R=r-x\right\} \mathrm{d} x
\end{aligned}
$$

Take Laplace transform on both sides of equation (8) with respect to $r$ over $(l,+\infty)$, we have

$$
\begin{aligned}
& \int_{l}^{+\infty} \operatorname{Pr}\left\{L_{m} \leq l \mid R=r\right\} e^{-s r} \mathrm{~d} r \\
= & \int_{l}^{+\infty} \int_{0}^{l} \lambda e^{-\lambda x} \operatorname{Pr}\left\{L_{m} \leq l \mid R=r-x\right\} e^{-s r} \mathrm{~d} x \mathrm{~d} r \\
= & \int_{0}^{l} \lambda e^{-\lambda x}\left(\int_{l}^{l+x} \operatorname{Pr}\left\{L_{m} \leq l \mid R=r-x\right\} e^{-s r} \mathrm{~d} r\right. \\
& \left.+\int_{l+x}^{+\infty} \operatorname{Pr}\left\{L_{m} \leq l \mid R=r-x\right\} e^{-s r} \mathrm{~d} r\right) \mathrm{d} x \\
= & \frac{e^{-s l}}{s}\left(1-e^{-\lambda l}\right)-\frac{\lambda e^{-s l}}{s(\lambda+s)}\left(1-e^{-(\lambda+s) l}\right) \\
& +\frac{\lambda}{\lambda+s}\left(1-e^{-(\lambda+s) l}\right)\left[G(l, s)-\frac{1-e^{-s l}}{s}\right] .
\end{aligned}
$$

Note that we also have

$$
\int_{l}^{+\infty} \operatorname{Pr}\left\{L_{m} \leq l \mid R=r\right\} e^{-s r} \mathrm{~d} r=G(l, s)-\frac{1-e^{-s l}}{s} .
$$

Following (9) and (10), we get

$$
G(l, s)=\frac{1-e^{-(\lambda+s) l}}{s+\lambda e^{-(\lambda+s) l}} .
$$

We have the following corollary about the region of convergence (ROC) of $G(l, s)$.

Corollary 3.2. The ROC of $G(l, s)$ includes the imaginary axis. More specifically, $g(l, r)$ is absolutely integrable, i.e.,

$$
\int_{-\infty}^{+\infty}|g(l, r)| d r<+\infty .
$$

Proof. See [12].

Following Theorem 3.1 and Corollary 3.2, the conditional CDF of $L_{m}$ given $R=r$ can be computed numerically by calculating the inverse Fourier Transform (FT) of $G(l, j \omega)$. The conditional CDFs of $L_{m}$ for selected $r$ 's are plotted in Fig. 2.

Remark 2. To obtain a closed form expression for the CDF of $L_{m}$, instead of fixing the routing distance $R=r$, we can model $R$ as an exponentially distributed RV of mean $\frac{1}{v}$. Based on Theorem 3.1, the CDF of $L_{m}$ can be calculated as

$$
\operatorname{Pr}\left\{L_{m} \leq l\right\}=\frac{v\left(1-e^{-(\lambda+v) l}\right)}{v+\lambda e^{-(\lambda+v) l}},
$$

for $l \geq 0$ 


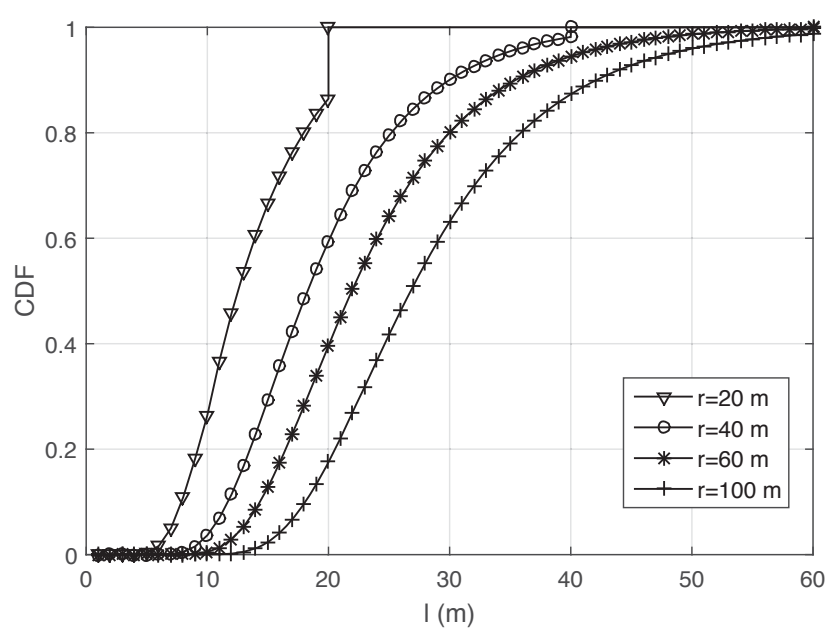

Figure 2: The conditional CDF $\operatorname{Pr}\left\{L_{m} \leq l \mid R=r\right\}$, where relay intensity $\lambda=0.1 / \mathrm{m}$.

Based on Theorem 3.1, we can also evaluate the mean and variance of $L_{m}$ with respect to the routing distance.

TheOREM 3.3. For a fixed relay intensity $\lambda$, as $r$ approaches $+\infty$, $\mathbb{E}\left\{L_{m} \mid R=r\right\} \sim O(\ln (r))$. More specifically, for $r>0$, let $m_{L_{m}}(r) \triangleq$ $\mathbb{E}\left\{L_{m} \mid R=r\right\}$, we have

$$
m_{L_{m}}(r)=\int_{0}^{r} \frac{1-e^{-\lambda x}}{\lambda x} d x,
$$

whose LT is

$$
M_{L_{m}}(s)=\frac{1}{s \lambda} \ln \left(\frac{\lambda}{s}+1\right) .
$$

Moreover, for any given $\lambda$, the conditional variance of $L_{m}$ under $R=r$, $\mathbb{D}\left\{L_{m} \mid R=r\right\}$, satisfies

$$
\lim _{r \rightarrow \infty} \mathbb{D}\left\{L_{m} \mid R=r\right\}=\frac{\pi^{2}}{6 \lambda^{2}} .
$$

Proof. See [12].

For a fixed relay intensity $\lambda$, the conditional mean of the longest hop distance $\mathbb{E}\left\{L_{m} \mid R=r\right\} \sim O(\ln (r))$ as the routing distance $r \rightarrow+\infty$, and its variance is bounded. Unlike the case with evenly deployed relays where the per hop distance stays constant with respect to the routing distance, for randomly distributed relays, the longest hop distance would goes to infinity as routing distance approaches infinity, i.e., the throughput of the worst hop would approach zero. This demonstrates that long distance communication is not feasible in randomly deployed networks.

\section{THE AVERAGE END-TO-END THROUGHPUT}

In this section, we derive the mean of the end-to-end throughput based on the distribution of the longest hop distance $L_{m}$ obtained in the previous section.

Given the coverage probability function $P_{S}(\cdot)$, the average coverage probability of the longest hop, $\mathbb{E}\left\{P_{s}\left(L_{m}\right)\right\}$, should only depend on the intensity of the relays, $\lambda$, and the routing distance $R$. For this reason, we define $p(x, r) \triangleq \mathbb{E}\left\{P_{s}\left(L_{m}\right) \mid \lambda=x, R=r\right\}$. Then, we have the following theorem on the end-to-end throughput.

THEOREM 4.1. Given the relay intensity $\lambda$ and routing distance $R$, the mean of the end-to-end throughput is given by

$$
\mathbb{E}\left\{T_{\text {end }}\right\}=\frac{e^{-\lambda R}}{\lambda} \int_{0}^{\lambda} e^{R x} p(x, R) d x .
$$

Proof. Recall that $\Phi$ is the PPP of the relays over $(0, R)$. The number of relays, $N$, is a random variable following Poisson distribution of mean $\lambda R$. Given the value of $N$, the relays are uniformly distributed over $(0, R)$. Denote the longest hop distance given $N=n$ by $L_{m}(n)$. The average end-to-end throughput can be expressed as

$$
\begin{aligned}
\mathbb{E}\left\{T_{\text {end }}\right\} & =\sum_{n=0}^{+\infty} e^{-\lambda R} \frac{(\lambda R)^{n}}{n !} \mathbb{E}\left\{\frac{1}{N+1} P_{s}\left(L_{m}\right) \mid N=n\right\} \\
& =\sum_{n=0}^{+\infty} e^{-\lambda R} \frac{(\lambda R)^{n}}{(n+1) !} \mathbb{E}\left\{P_{s}\left(L_{m}(n)\right)\right\}
\end{aligned}
$$

Note that $L_{m}(n)$ is the longest hop distance for $n$ uniformly distributed relays over $(0, R)$. Define $p_{n}(r) \triangleq \mathbb{E}\left\{P_{s}\left(L_{m}(n)\right) \mid R=r\right\}$, then we have

$$
\mathbb{E}\left\{T_{\text {end }}\right\}=\sum_{n=0}^{+\infty} e^{-\lambda R} \frac{(\lambda R)^{n}}{(n+1) !} p_{n}(R) .
$$

Moreover, note that

$$
p(x, r)=\sum_{n=0}^{+\infty} e^{-x r} \frac{(x r)^{n}}{n !} p_{n}(r) .
$$

For routing distance $R$, define

$$
h(t) \triangleq \sum_{n=0}^{+\infty} \frac{t^{(n+1)}}{(n+1) !} p_{n}(R) .
$$

The derivative of $h(t)$ can be calculated as

$$
h^{\prime}(t)=\sum_{n=0}^{+\infty} \frac{t^{n}}{n !} p_{n}(R)=e^{t} p\left(\frac{t}{R}, R\right) .
$$

It then follows that

$$
h(t)=\int_{0}^{t} h^{\prime}(u) \mathrm{d} u=\int_{0}^{t} e^{u} p\left(\frac{u}{R}, R\right) \mathrm{d} u,
$$

and

$$
\mathbb{E}\left\{T_{\text {end }}\right\}=\frac{e^{-\lambda R}}{\lambda R} h(\lambda R)=\frac{e^{-\lambda R}}{\lambda R} \int_{0}^{\lambda R} e^{u} p\left(\frac{u}{R}, R\right) \mathrm{d} u .
$$

Let $x=\frac{u}{R}$, then it follows from (17) that

$$
\mathbb{E}\left\{T_{\text {end }}\right\}=\frac{e^{-\lambda R}}{\lambda} \int_{0}^{\lambda} e^{R x} p(x, R) \mathrm{d} x .
$$

This completes the proof.

Note that function $p(x, r)$ can be computed numerically, which only depends on the marginal distribution of $L_{m}$. Following Theorem 4.1, we can calculate $\mathbb{E}\left\{T_{\text {end }}\right\}$ without deriving the joint probability density function (PDF) of $N$ and $L_{m}$ explicitly. With the following Lemma, we can further reduce the computational complexity by calculating the Laplace transform of $\mathbb{E}\left\{T_{\text {end }}\right\}$ with respect to the routing distance. 
LEMMA 4.2. Taking the relay intensity $\Lambda$ as a random variable and let $f_{L_{m} \mid \Lambda, R}(l \mid x, r)$ denote the conditional PDF of the longest hop distance $L_{m}$ given relay intensity $\Lambda=x$ and routing distance $R=r$. Define

$$
q(l, \lambda, r) \triangleq \frac{e^{-\lambda r}}{\lambda} \int_{0}^{\lambda} e^{r x} f_{L_{m} \mid \Lambda, R}(l \mid x, r) d x,
$$

then the Laplace transform of $q(l, \lambda, r)$ with respect to $r$ is

$$
Q(l, \lambda, s)=\frac{(s+\lambda)}{\lambda+e^{(s+\lambda) l} s}
$$

This follows directly from the Laplace transform of $f_{L_{m} \mid \Lambda, R}(l \mid$ $x, r)$ in (??), we skip the proof for brevity. Based on Lemma 4.2, we have the following result on $\mathbb{E}\left\{T_{\text {end }}\right\}$.

Proposition 4.3. For a fixed relay intensity $\lambda$, define $T_{\text {end }}(r) \triangleq$ $\mathbb{E}\left\{T_{\text {end }} \mid R=r\right\}$ as the average end-to-end throughput given routing distance $R=r$. The Laplace transform of $T_{\text {end }}(r), \mathcal{T}_{\text {end }}(s)$, can be calculated as

$$
\mathcal{T}_{\text {end }}(s)=\int_{0}^{+\infty} P_{s}(l) \frac{s+\lambda}{\lambda+e^{(s+\lambda) l} s} d l .
$$

Proof. Recall that $p(x, r)=\mathbb{E}\left\{P_{s}\left(L_{m}\right) \mid \lambda=x, R=r\right\}$, where $P_{s}\left(L_{m}\right)$ is the coverage probability of the longest hop, then $p(x, r)$ can be calculated as

$$
p(x, r)=\int_{0}^{+\infty} P_{s}(l) f_{L_{m} \mid \Lambda, R}(l \mid x, r) \mathrm{d} l .
$$

So $T_{\text {end }}(r)$ can be expressed as

$$
\begin{aligned}
T_{\text {end }}(r) & =\frac{e^{-\lambda r}}{\lambda} \int_{0}^{\lambda} e^{r x} \int_{0}^{+\infty} P_{s}(l) f_{L_{m} \mid \Lambda, R}(l \mid x, r) \mathrm{d} l \mathrm{~d} x \\
& =\int_{0}^{+\infty} P_{s}(l) q(l, \lambda, r) \mathrm{d} l .
\end{aligned}
$$

Based on Lemma 4.2, (20) can be obtained accordingly.

Following Proposition 4.3 and Corollary 3.2, the mean of the throughput can be computed numerically through the inverse Fourier transform of $T_{\text {end }}(j \omega)$.

As is shown in Proposition 4.3, a closed-form expression of the end-to-end throughput is hard to derive. In order to further analyze the impacts of different network parameters on network performance, we derive the following lower bound on end-to-end throughput.

Proposition 4.4. For a given relay intensity $\lambda$ and routing distance $r$, the end-to-end throughput $T_{\text {end }}(r)$ is lower bounded by $T_{\text {end,L }}(r)$, i.e., $T_{\text {end }}(r) \geq T_{\text {end,L }}(r)$, where

$$
\begin{aligned}
T_{\text {end }, L}(r)= & \frac{1-e^{-\lambda r}}{\lambda r} \exp \left(-\frac{\kappa \lambda^{-2}}{1-e^{-\lambda r}}\left[\frac{\ln ^{2}(\lambda r)}{2}+B(\lambda r) \ln (\lambda r)\right.\right. \\
& \left.\left.+C(\lambda r)+c-e^{-\lambda r}\left(A(\lambda r)^{2}+(2+c) \lambda r+c\right)\right]\right),
\end{aligned}
$$

with $c=\max _{t \in(0,1)} \frac{\ln ^{2} t}{(1-t)^{2}}-\ln ^{2} t \approx 1.51, A \triangleq \int_{0}^{+\infty} \frac{l^{2} e^{-l}}{1-e^{-l}} d l \approx$ $2.404, B(x) \triangleq \int_{0}^{1} \frac{1-e^{-u}}{u} d u-\int_{1}^{x} \frac{e^{-u}}{u} d u, C(x) \triangleq \int_{1}^{x} \ln u \frac{e^{-u}}{u} d u-$ $\int_{0}^{1} \ln u \frac{1-e^{-u}}{u} d u$.

For $\lambda r \gg 1$, the lower bound $T_{\text {end,L }}(r)$ approximates

$$
T_{\text {end, } L}(r) \approx \frac{1}{\lambda r} \exp \left(-\kappa \lambda^{-2}\left[\frac{\ln ^{2}(\lambda r)}{2}+B \ln (\lambda r)+C+c\right]\right),
$$

where $B=\lim _{x \rightarrow+\infty} B(x) \approx 0.577, C=\lim _{x \rightarrow+\infty} C(x) \approx 0.989$.

Proof. See [12].

Note that a closed-form expression for the end-to-end throughput is difficult to obtain, in the following we consider to optimize the lower bound $T_{\text {end,L }}(r)$ with respect to the relay intensity $\lambda$, and obtain the following result.

Corollary 4.5. For $\lambda r \gg 1$, the optimal relay intensity $\lambda^{*}$ that maximizes $T_{\text {end,L }}(r)$ should satisfy $\lambda_{1}<\lambda^{*}<\lambda_{2}$, where

$$
\begin{gathered}
\lambda_{1}=\frac{1}{r} \exp \left(\frac{1}{r \sqrt{\kappa}} \exp \left(-W_{-1}\left(-\frac{1}{r \sqrt{\kappa}}\right)\right),\right. \\
\lambda_{2}=\frac{1}{r} \exp \left(\frac{e^{-\sqrt{2 C+2 c-B}}}{r \sqrt{\kappa}} \exp \left(-W_{-1}\left(-\frac{e^{-\sqrt{2 C+2 c-B}}}{r \sqrt{\kappa}}\right)\right),\right.
\end{gathered}
$$

where $W_{-1}(\cdot)$ is the real branch of Lambert $W$ function over $(-\infty,-1)[6]$.

Proof. See [12]

Remark 3. As discussed earlier, if we model $R$ as an exponentially distributed RV with mean $\frac{1}{v}$, the mean of the throughput can be obtained as

$$
\mathbb{E}\left\{T_{\text {end }}\right\}=\int_{0}^{+\infty} P_{s}(l) \frac{v(v+\lambda)}{\lambda+e^{(v+\lambda) l} v} \mathrm{~d} l
$$

Note that

$$
\frac{\partial\left(\frac{v(v+\lambda)}{\lambda+e^{(v+\lambda) l} v}\right)}{\partial \lambda}=v^{2} \frac{[1-(v+\lambda) l] e^{(v+\lambda) l}-1}{\left(\lambda+e^{(v+\lambda) l} v\right)^{2}},
$$

which is negative for all $(v+\lambda) l>0$. So in this case, $\mathbb{E}\left\{T_{\text {end }}\right\}$ is a decreasing function with respect to $\lambda$. That is, as the relay intensity increases, the randomly deployed relays may degrade, rather than improve the throughput performance of the system. The underlying argument is that if the routing distance is very short, nearest neighbor relaying is more likely a burden rather than a necessary step when taking the extra spectrum and power it consumes into account. At the same time, when the routing distance is exponentially distributed, the probability of having a short routing distance is very high.

The nearest neighbor routing protocol aims to guarantee the link quality of each single hop by utilizing all the available relays in $\Phi$ and minimizing the hop distances. When the node intensity $\lambda$ is large, the nearest neighbor routing protocol will degrade the end-to-end throughput because of the extra delay and bandwidth it takes. In this case, we can select $\Phi^{\prime} \subset \Phi$, which is a thinning of $\Phi$ and a HPPP of intensity $\lambda^{*}$ with $\lambda^{*}<\lambda$. We can then apply the nearest neighbor routing protocol over $\Phi^{\prime}$ rather than $\Phi$. Please refer to Corollary 4.5 for the selection of the optimal relay intensity $\lambda^{*}$.

\section{NUMERICAL RESULTS}

In this section, we evaluate the end-to-end throughput performance with random relay deployment through numerical results. Unless otherwise clarified, we will use the following parameters: the interferer intensity $\mu=5 \times 10^{-4} / \mathrm{m}^{2}$, the ALOHA access probability $p_{a}=0.1$, the path-loss exponent $\beta=4$, the SINR threshold for successful transmission $\theta=10 \mathrm{~dB}$. 


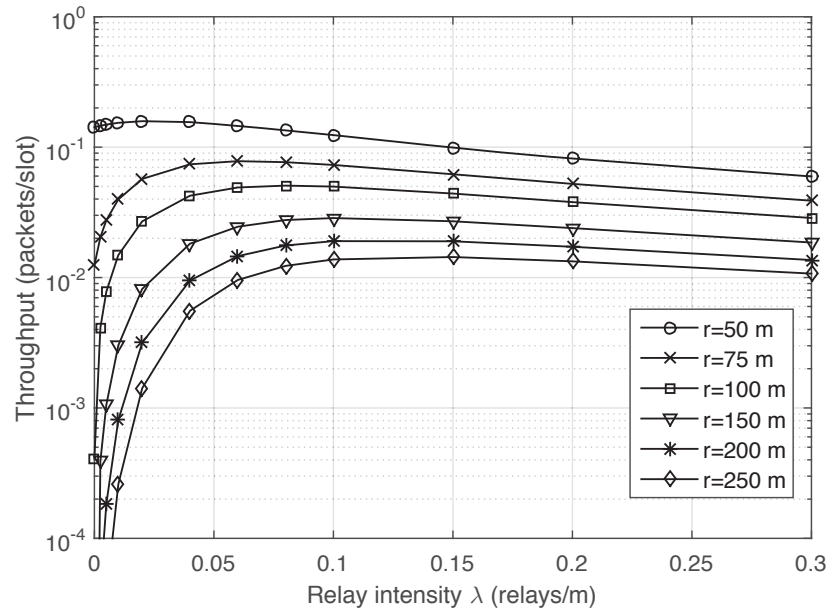

Figure 3: Average end-to-end throughput versus relay intensity under different routing distances.

Example 1: End-to-end throuhgput with random relay deployment. In this example, we evaluate the end-to-end throughput with random relays. Fig. 3 shows the average end-to-end throughput versus relay intensity $\lambda$ for different routing distance $r$ with random relay deployment. It can be observed that: even without an optimized deployment of relays, the end-to-end throughput can still be obviously improved compared with the case of direct connection. For example, if a minimum end-to-end throughput of $1 \times 10^{-2}$ packets/slot is required, the maximum communication range is around $75 \mathrm{~m}$ without the relays, while the communication range expands to more than $250 \mathrm{~m}$ with multi-hop relays. To improve the end-to-end throughput, we can optimize the relay intensity for different routing distance. Instead of using the NN routing, source can select relays by thinning the original PP of relays to the optimal intensity basing on the routing distance. It can also be observed that the optimal relay intensity $\lambda$ increases as $r$ increases, on the contrary to the equidistant relays where the optimal relay distance stays constant ${ }^{2}$.

Example 2: Performance comparison between equidistant and random relays. In this example, we compare the end-to-end throughput between equidistant and random relays. Fig. 4 shows the optimal end-to-end throughput of random relay deployment and that of equidistant relays under different routing distance $r$ and SINR threshold $\theta$. The relay intensity $\lambda$ is optimized for each routing distance. The random relay deployment suffers a significant performance loss compared to the ideal case. For instance, with a SINR threshold $\theta$ of $10 \mathrm{~dB}$ under the network configuration, there is a $48 \%$ throughput loss at $r=50 \mathrm{~m}$ and a $70 \%$ performance loss at $r=130 \mathrm{~m}$, which are not negligible for system evaluation.

\section{CONCLUSION \& DISCUSSION}

In this paper, we investigated the effect of relay randomness on the end-to-end throughput in multi-hop wireless networks using

\footnotetext{
${ }^{2}$ Here, we refer to the upper bound of end-to-end throughput for equidistant relays, $\frac{d}{r} P_{\text {cov }}(d)$, where $d$ is the per hop distance, and the optimal $d$ is independent of $r$.
}

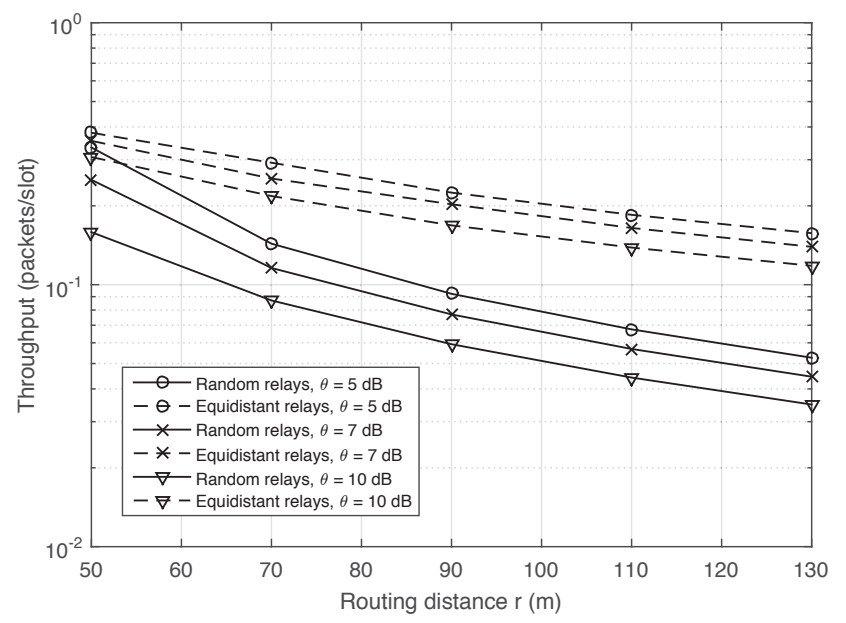

Figure 4: Throughput comparison under TDMA: random relays versus equidistant relays

stochastic geometry. We modeled the relays as a linear Poisson Point Process between the source and destination, and the external interferers as an independent Poisson Point Process. Assuming the nearest neighbor routing protocol, we evaluated the end-to-end throughput. Based on the throughput analysis, we derived the range of the optimal relay density for any given routing distance and node density. The analysis was further demonstrated through numerical examples. Both the theoretic and numerical results indicated that: (i) Compared with the relay-free case, random distributed relay can significantly extend the communication distance, even without a refined deployment; (ii) Systems with equidistant relays generally achieve much higher throughput than those with random relays; (iii) Optimal relay intensity varies with the routing distance, node density and the interference levels. (iv) Long distance communication is not feasible with random relays. Optimal routing protocol design for randomly located relays will be further explored in our future research.

\section{REFERENCES}

[1] J.G. Andrews, F. Baccelli, and R.K. Ganti. 2011. A Tractable Approach to Coverage and Rate in Cellular Networks. IEEE Transactions on Communications 59, 11 (November 2011), 3122-3134. DOI : http://dx.doi.org/10.1109/TCOMM.2011. 100411.100541

[2] J. G. Andrews, S. Weber, M. Kountouris, and M. Haenggi. 2010. Random access transport capacity. IEEE Transactions on Wireless Communications 9, 6 (June 2010), 2101-2111. DOI : http://dx.doi.org/10.1109/TWC.2010.06.091432

[3] François Baccelli and Bartlomiej Blaszczyszyn. 2009. Stochastic Geometry and Wireless Networks, Volume 1: Theory. Foundations and Trends in Networking 3, 3-4 (2009), 249-449. DOI : http://dx.doi.org/10.1561/1300000006

[4] F. Baccelli, B. Blaszczyszyn, and P. Muhlethaler. 2006. An Aloha protocol for multihop mobile wireless networks. IEEE Transactions on Information Theory 52, 2 (Feb 2006), 421-436. DOI : http://dx.doi.org/10.1109/TIT.2005.862098

[5] B. Blaszczyszyn and P. Mühlethaler. 2015. Random Linear Multihop Relaying in a General Field of Interferers Using Spatial Aloha. IEEE Transactions on Wireless Communications 14, 7 (July 2015), 3700-3714. DOI : http://dx.doi.org/10.1109/ TWC.2015.2409845

[6] R. M. Corless, G. H. Gonnet, D. E. G. Hare, D. J. Jeffrey, and D. E. Knuth. 1996 On the LambertW function. Advances in Computational Mathematics 5, 1 (1996), 329-359. DOI : http://dx.doi.org/10.1007/BF02124750

[7] H. ElSawy, E. Hossain, and M. Haenggi. 2013. Stochastic Geometry for Modeling, Analysis, and Design of Multi-Tier and Cognitive Cellular Wireless Networks: A Survey. IEEE Communications Surveys Tutorials 15, 3 (Thirdquarter 2013), 
996-1019. DOI : http://dx.doi.org/10.1109/SURV.2013.052213.00000

[8] H. Feng and L. J. Cimini. 2012. On the optimum number of hops in a multihop linear network with randomly located nodes. In 2012 IEEE International Conference on Communications (ICC). 2329-2333. DOI : http://dx.doi.org/10.1109/ ICC. 2012.6363752

[9] Martin Haenggi. 2012. Stochastic Geometry for Wireless Networks (1st ed.). Cambridge University Press, New York, NY, USA.

[10] M. Haenggi, J.G. Andrews, F. Baccelli, O. Dousse, and M. Franceschetti. 2009 Stochastic geometry and random graphs for the analysis and design of wireless networks. IEEE fournal on Selected Areas in Communications 27, 7 (September 2009), 1029-1046. DOI : http://dx.doi.org/10.1109/JSAC.2009.090902

[11] Martin Haenggi and Radha Krishna Ganti. 2009. Interference in Large Wireless Networks. Foundations and Trends in Networking 3, 2 (February 2009), 127-248. DOI: http://dx.doi.org/10.1561/1300000015

[12] Y. Liang and T. Li. 2017. End-to-End Throughput in Multi-Hop Wireless Networks With Random Relay Deployment. (2017). In preparation.

[13] M. Sikora, J. N. Laneman, M. Haenggi, D. J. Costello, and T. E. Fuja. 2006. Bandwidth- and power-efficient routing in linear wireless networks. IEEE Transactions on Information Theory 52, 6 (June 2006), 2624-2633. DOI : http: //dx.doi.org/10.1109/TIT.2006.874520

[14] E. S. Sousa and J. A. Silvester. 1990. Optimum transmission ranges in a directsequence spread-spectrum multihop packet radio network. IEEE fournal on Selected Areas in Communications 8, 5 (Jun 1990), 762-771. DOI : http://dx.doi. org $/ 10.1109 / 49.56383$

[15] K. Stamatiou and M. Haenggi. 2010. The delay-optimal number of hops in Poisson multi-hop networks. In 2010 IEEE International Symposium on Information Theory. 1733-1737. DOI : http://dx.doi.org/10.1109/ISIT.2010.5513261

[16] K. Stamatiou and M. Haenggi. 2014. Delay Characterization of Multihop Transmission in a Poisson Field of Interference. IEEE/ACM Transactions on Networking 22, 6 (Dec 2014), 1794-1807. DOI : http://dx.doi.org/10.1109/TNET.2013.2283338

[17] S.P. Weber, Xiangying Yang, J. G. Andrews, and G. de Veciana. 2005. Transmission capacity of wireless ad hoc networks with outage constraints. IEEE Transactions on Information Theory 51, 12 (Dec 2005), 4091-4102. DOI : http://dx.doi.org/10. 1109/TIT.2005.858939 